\title{
Gasto energético e medidas antropométricas de novas usuárias do contraceptivo injetável trimestral de acetato de medroxiprogesterona de depósito
}

\author{
Energy expenditure and anthropometric \\ measurements in new users of \\ depot medroxyprogesterone
}

acetate

Priscilla de Nazaré Silva dos SANTOS ${ }^{1}$

Marina SIDER²

Waleska Oliveira MODESTO ${ }^{1}$

Maria Valeria BAHAMONDES 3

Luis BAHAMONDES ${ }^{3}$

Arlete FERNANDES ${ }^{3}$

\section{R E S U M O}

\section{Objetivo}

Avaliar o gasto energético e as medidas antropométricas de mulheres durante o primeiro ano de uso do método contraceptivo de acetato de medroxiprogesterona de depósito.

\section{Métodos}

Estudo prospectivo com grupo de comparação. Foram incluídas mulheres saudáveis, não obesas, nunca usuárias de acetato de medroxiprogesterona de depósito e sem antecedentes que pudessem contribuir para a variação do peso corporal; foram distribuídas em dois grupos, 28 usuárias de acetato de medroxiprogesterona e 24 usuárias de dispositivo intrauterino de cobre, pareadas por idade $\left( \pm 1\right.$ ano) e índice de massa corporal $\left(\mathrm{kg} / \mathrm{m}^{2}\right)$.

\footnotetext{
1 Universidade Estadual de Campinas, Faculdade de Ciências Médicas, Departamento de Tocoginecologia. Campinas, SP, Brasil.

2 Médica. Campinas, SP, Brasil.

3 Universidade de Campinas, Faculdade de Ciências Médicas, Departamento de Obstetrícia e Ginecologia. R. Tessália Vieira de Camargo, 126, Cidade Universitária Zeferino Vaz, 13083-887, Campinas, SP, Brasil. Correspondência para/Correspondence to: A FERNANDES. E-mail: <arlete@fcm.unicamp.br>.

Apoio: Conselho Nacional de Desenvolvimento Científico e Tecnológico, Fundação de Amparo à Pesquisa do Estado de São Paulo e Secretaria de Saúde do Estado de São Paulo (\#2009/53293-0).
} 
As variáveis estudadas foram sociodemográficas (idade, etnia, tabagismo, etilismo, atividade física, classe econômica e escolaridade), peso $(\mathrm{kg})$, índice de massa corporal, gasto energético basal e total, medidas de circunferência de cintura e quadril $(\mathrm{cm})$ e relação cintura-quadril.

\section{Resultados}

A idade das mulheres variou de 20-39 anos. As médias de idade/índice de massa corporal foram $29,6(\mathrm{DP}= \pm 5,2)$ anos $/ 23,9\left( \pm 3,6 \mathrm{~kg} / \mathrm{m}^{2}\right)$, no grupo de acetato de medroxiprogesterona de depósito, e de $28,6(\mathrm{DP}= \pm 5,2)$ anos/ $24,5\left( \pm 2,7 \mathrm{~kg} / \mathrm{m}^{2}\right)$, no grupo de dispositivo intrauterino de cobre. Após análise de variância para medidas repetidas, as usuárias de acetato de medroxiprogesterona de depósito apresentaram ganho de 2,2 kg no peso corporal e de -0,2 kg no grupo do dispositivo intrauterino de cobre, sem diferença estatisticamente significativa entre eles. Não houve discrepância nas demais variáveis estudadas.

\section{Conclusão}

Mulheres saudáveis e jovens não apresentaram mudança no peso, nas medidas e nos gastos energéticos durante o primeiro ano de uso do contraceptivo acetato de medroxiprogesterona. A orientação em relação aos hábitos saudáveis de vida e o monitoramento de medidas são importantes para o controle do peso corporal em usuárias de métodos contraceptivos.

Palavras-chave: Antropometria. Contracepção. Gasto energético. Medroxiprogesterona. Peso corporal.

\section{A B S T R A C T}

\section{Objective}

The objective of this study was to assess energy expenditure and the anthropometric profile of women during the first year of use of depot medroxyprogesterone acetate contraception.

\section{Methods}

This prospective study included healthy non-obese women who had never used depot-medroxyprogesterone acetate and did not have a history of weight fluctuations. The women were divided into two groups composed of 28 depot medroxyprogesterone acetate users and 24 copper intrauterine device (TCu380A) users. They were paired for age $\left( \pm 1\right.$ year) and body mass index $\left( \pm 1 \mathrm{~kg} / \mathrm{m}^{2}\right)$. The following variables were used: sociodemographic characteristics (age, ethnicity, smoking status, alcohol consumption, physical activity, economic class, and education level), weight ( $\mathrm{kg}$ ), body max index, resting and total energy expenditure, waist and hip circumferences $(\mathrm{cm})$, and waist-to-hip ratio.

\section{Results}

The age of the women studied ranged from 20-39 years. The mean values of age/body mass index ratio were $29.6(S D= \pm 5.2)$ years $/ 23.9\left( \pm 3.6 \mathrm{~kg} / \mathrm{m}^{2}\right)$ in the depot medroxyprogesterone acetate group and $28.6(S D= \pm 5.2)$ years $/ 24.5\left( \pm 2.7 \mathrm{~kg} / \mathrm{m}^{2}\right)$ in the intrauterine device group. After conducting repeated measures analysis of variance, the users of depot medroxyprogesterone acetate showed weight gain of $2.2 \mathrm{~kg}$, and those in the intrauterine device group showed weight loss of $0.2 \mathrm{~kg}$ without statistically significant difference between the groups. There were no significant differences between the other variables.

\section{Conclusion}

There were no changes in weight, anthropometric measurements, and energy expenditure in the young and healthy women during the first year of use of depot medroxyprogesterone acetate contraception. Guidelines and recommendations for a healthy lifestyle to avoid changes in the anthropometric measurements are important for weight control in users of contraceptive methods.

Keywords: Antropometry. Contraception. Energy metabolism. Medroxyprogesterone. Body weight.

\section{N T R O D U Ç Ã O}

O Acetato de Medroxiprogesterona de Depósito (AMPD) é um contraceptivo de alta eficácia e segurança utilizado por mulheres em todo o mundo há mais de seis décadas'. Pela facilidade de uso, com uma injeção trimestral intramuscular, e baixo custo, o método tem sido adotado por mulheres jovens e adolescentes e distribuído pelo setor público em diferentes países ${ }^{2,3}$. 
Entretanto, inúmeras pesquisas têm sido dedicadas a avaliar a variação do peso em mulheres usuárias de AMPD com resultados controversos $^{4-6}$. As taxas de descontinuação de uso do método, atribuídas ao ganho de peso, têm sido descritas em torno de 2 a $5 \%$ entre as usuárias ${ }^{7}$.

Com base em estudos prospectivos com diferentes tempos de observação é possível afirmar que algumas mulheres adultas e não obesas ganham peso excessivo a partir do início do uso de AMPD, à custa de depósitos de gordura ${ }^{5,6,8,9}$. Algumas pesquisas têm focado a variação de peso em usuárias de AMPD em relação à composição corporal e utilizado grupos de comparação com usuárias de outros métodos hormonais e não hormonais, apresentando resultados de maior concentração central de depósitos de gordura em usuárias de $\mathrm{AMPD}{ }^{5,9}$.

Em estudo placebo-controlado, randomizado e cego, o qual forneceu três refeições diárias padronizadas durante três meses a dez mulheres que utilizaram uma única dose de AMPD e as dez que utilizaram placebo, não foram observadas variações na ingestão alimentar, no gasto energético e no ganho de peso entre os grupos ${ }^{10}$. Em vista da falta de estudos clínicos para avaliar o metabolismo em usuárias do contraceptivo com maior tempo de observação, optamos por comparar duas coortes de mulheres, usuárias em início de uso do AMPD e de Dispositivo Intrauterino de Cobre (DIU), nos tempos basal e trimestralmente até 12 meses de seguimento.

\section{M É T O D O S}

Os critérios de inclusão foram idade de 18-40 anos, Índice de Massa Corporal (IMC) $<30 \mathrm{~kg} / \mathrm{m}^{2}$, teste de pós-carga de $75 \mathrm{mg}$ de glicose via oral, com glicemia de jejum $<100 \mathrm{mg} \%$ e de 120 '<140 mg\%. Foram excluídas mulheres em período de aleitamento, com antecedente familiar de primeiro grau com diabete Mellitus, portadoras de diabete Mellitus tipos 1 e 2, síndrome plurimetabólica, hipertensão arterial sistêmica, hiper e hipotiroidismo, insuficiência renal crônica e transplantadas de qualquer órgão, mulheres em uso de medicamentos (como corticoesteróides, antipsicóticos, tiazídicos e estatinas), com sinais de hirsutismo e/ou hiperandrogenismo, acantose nigricans, síndrome do ovário policístico, antecedente de cirurgia bariátrica ou mulheres que já tivessem utilizado AMPD em algum momento da vida reprodutiva.

A pesquisa foi veiculada nas Unidades Básicas de Saúde e compareceram 290 mulheres das quais somente 72 cumpriram os critérios de inclusão, sendo que, destas últimas, 20 desistiram antes do início ou nos primeiros três meses da pesquisa. Foram incluídas neste estudo 54 mulheres pareadas por idade $\left( \pm 1\right.$ ano) e IMC $\left( \pm 1 \mathrm{~kg} / \mathrm{m}^{2}\right)$ as quais puderam escolher o método contraceptivo, o AMPD (Contracept ${ }^{\circledR}$, EMS, Hortolândia, São Paulo) ou o DIU de cobre 380A (Óptima, Injeflex, São Paulo [SP], TCu 380A).

Todas as participantes retornaram ao Serviço trimestralmente e, nessa ocasião, as usuárias de AMPD receberam administração de uma dose de 150 mg do contraceptivo via intramuscular. A cada retorno, todas as mulheres responderam a um questionário sobre hábitos de vida e tiveram aferidas as medidas de composição corporal pela mesma profissional. Todas foram estimuladas a incorporar hábitos saudáveis de alimentação e de atividade física aeróbica três vezes por semana.

As variáveis sociodemográficas e os hábitos de vida foram avaliados por meio de entrevista baseada no autorrelato. As variáveis estudadas foram idade, etnia (branca/não branca), escolaridade ( $\leq 8 />8$ anos) e classe econômica (A [R\$14.366,00-8.099,00], $B$ [R\$4.588,00-2.327,00], $C[R \$ 1.391,00-933,00]$ ou $D[R \$ 618,00])^{11}$. As de hábitos de vida foram: tabagismo (sim: fumante [ $\geq 100$ cigarros na vida] ou não: ex-fumante $[<100$ cigarros na vida] ou não fumante) ${ }^{12}$, consumo de álcool ( $\leq 1$ dose/>1 dose por mês, de acordo com a ingestão de dose [cerveja, $300 \mathrm{~mL}$; vinho, $120 \mathrm{~mL}$; destilado, $30 \mathrm{~mL}])^{12}$ e atividade física (ativa: atividade física aeróbica $\geq 150$ minutos por sema- 
na/não ativa: sem atividade ou $\leq 150$ minutos de atividade física aeróbica por semana $)^{13}$. As medidas antropométricas de peso (kg), altura (cm), IMC e medidas de circunferências da cintura, do quadril $(\mathrm{cm})$ e relação cintura-quadril foram aferidas por meio de fita métrica inextensível ${ }^{14}$.

O Gasto Energético Basal (GEB) e Total (GET) foi calculado segundo fórmula de Harris-Benedict, validada e aplicável na prática clínica em diferentes idades e tipos corporais ${ }^{15}$. O valor de cálculo do GEB foi ajustado para sexo, peso $(\mathrm{kg})$, altura (cm) e idade (anos) e, para a obtenção da GET em pessoas ativas, multiplicou-se o GEB por 1,3 de fator de atividade ${ }^{16}$.

O tamanho amostral foi baseado nas diferenças descritas em estudo anterior ${ }^{9} \mathrm{de}+1,7$ e $-0,3 \%$ do percentual de gordura total encontradas em usuárias de AMPD e de DIU de cobre, respectivamente, após 12 meses de uso do método. Para a análise de variância nas medidas repetidas em amostra pareada por idade $( \pm 1)$ e IMC $( \pm 1)$, considerando um nível de significância de $5 \%$ e poder de $80 \%$, foi requerido um número de 20 mulheres em cada grupo ${ }^{17}$.

Os dados categóricos foram comparados utilizando o teste Qui-quadrado ou teste exato de Fisher e as médias pelo de Mann-Whitney. Foi utilizado o teste de Análise de Variância (Anova) para comparar as médias das medidas repetidas. O nível de significância estabelecido foi de $5 \%$ e poder de teste de $80 \%$ e o programa usado para as análises foi o Statistical Analysis System (SAS, Institute Inc., 2002-2008, Cary, Carolina do Norte, Estados Unidos) versão 9.3.

Este estudo foi realizado na Unidade de Reprodução Humana do Departamento de Tocoginecologia da Faculdade de Ciências Médicas da Universidade Estadual de Campinas, no período de fevereiro de 2011 a fevereiro de 2013, e faz parte de estudo registrado no <https://clinincaltrials.gov>, sob o número NCT01527526. O projeto obteve aprovação do Comitê de Ética em Pesquisa da Universidade (\#2009/53293-0) e todas as mulheres que aceitaram participar do estudo assinaram o Termo de Consentimento Livre e Esclarecido.

\section{RES U LTA D O S}

Das 54 mulheres que iniciaram o estudo, duas foram excluídas durante o seguimento, uma por apresentar osteoporose e outra por desenvolver hipotireoidismo. Foram avaliadas 52 mulheres durante 12 meses, 28 no grupo de AMPD e 24 no do DIU.

A idade das participantes variou de 20-39 anos, com média de 29,6 e 28,6 anos nos grupos de AMPD e DIU, respectivamente, e média de IMC de 23,9 e $24,5 \mathrm{~kg} / \mathrm{m}^{2}$, na mesma ordem de grupos. Em ambos, dois terços das mulheres eram não brancas e pertenciam às classes econômicas C e D; aproximadamente $80 \%$ eram não fumantes e não etilistas, e somente cerca de $30 \%$ realizavam exercício físico regular. As mulheres do grupo de AMPD tiveram mais anos de escolaridade em relação às do grupo do DIU. Não houve diferenças significativas entre os grupos no momento basal com relação às demais variáveis estudadas (Tabela 1).

Os hábitos de vida referidos pelas participantes a cada retorno trimestral estão mostrados na Tabela 2. Aos 12 meses foi observado aumento no número de usuárias de AMPD fisicamente ativas, embora sem diferença significativa entre os grupos (Tabela 2). Após Anova para medidas repetidas, não foram observadas mudanças significativas entre os grupos em relação às variáveis estudadas. O grupo de AMPD apresentou média de ganho de $2,2 \mathrm{~kg}$ no peso corporal enquanto o grupo do DIU, -0,2 kg aos 12 meses, sem diferença estatisticamente significativa entre os grupos (Tabela 3).

D I S C U S S Ã O

Este estudo, realizado com mulheres adultas saudáveis e idade inferior a 40 anos, não 
Tabela 1. Características antropométricas, sociodemográficas e de gastos energéticos dos grupos de Acetato de Medroxiprogesterona de Depósito (AMPD) e Dispositivo Intrauterino de Cobre (DIU) no início do estudo. Campinas (SP), 2011.

\begin{tabular}{|c|c|c|c|}
\hline Variáveis & AMPD $(n=28)$ & DIU $(n=24)$ & $p$-valor \\
\hline Idade em anos, média (DP) & $29,6(5,2)$ & $28,6(5,2)$ & $0,459^{\mathrm{a}}$ \\
\hline Peso (kg), média (DP) & $62,4(9,8)$ & $60,5(6,6)$ & $0,491^{a}$ \\
\hline Índice de massa corporal $\left(\mathrm{kg} / \mathrm{m}^{2}\right)$, média (DP) & $23,9(3,6)$ & $24,5(2,7)$ & $0,502^{\mathrm{a}}$ \\
\hline \multicolumn{4}{|l|}{ Etnia, $n(\%)$} \\
\hline Branca & $11(39,3)$ & $10(41,6)$ & \multirow[t]{2}{*}{$0,861^{b}$} \\
\hline Não branca & $17(60,7)$ & $14(58,3)$ & \\
\hline \multicolumn{4}{|l|}{ Classe econômica, n (\%) } \\
\hline A ou $B$ & $10(35,7)$ & $8(33,3)$ & \multirow[t]{2}{*}{$0,857^{\mathbf{b}}$} \\
\hline C ou D & $18(64,3)$ & $16(66,7)$ & \\
\hline \multicolumn{4}{|l|}{ Escolaridade, $n(\%)$} \\
\hline$\leq 8$ anos & $8(28,5)$ & $15(62,5)$ & \multirow[t]{2}{*}{$0,014^{b}$} \\
\hline$>8$ anos & $20(71,5)$ & $9(37,5)$ & \\
\hline \multicolumn{4}{|l|}{ Tabagismo, $n$ (\%) } \\
\hline Sim & $3(10,7)$ & $3(12,5)$ & \multirow[t]{2}{*}{$1,000^{c}$} \\
\hline Não & $25(89,3)$ & $21(87,5)$ & \\
\hline \multicolumn{4}{|l|}{ Etilismo, $n$ (\%) } \\
\hline$\leq 1$ dose & $23(82,1)$ & $19(79,1)$ & \multirow[t]{2}{*}{$1,000^{c}$} \\
\hline$>1$ dose & $5(17,9)$ & $5(20,9)$ & \\
\hline \multicolumn{4}{|l|}{ Atividade fisica, $n$ (\%) } \\
\hline Sim & $6(21,4)$ & $8(33,3)$ & \multirow[t]{2}{*}{$0,334^{b}$} \\
\hline Não & $22(78,6)$ & $16(66,7)$ & \\
\hline \multicolumn{4}{|l|}{ Gasto energético (kcal), média (DP) } \\
\hline Gasto energético basal & $1336,8(249,1)$ & $1413,1(66,8)$ & $0,474^{a}$ \\
\hline Gasto energético total & $1737,8(323,8)$ & $1837,0(86,9)$ & $0,474^{a}$ \\
\hline
\end{tabular}

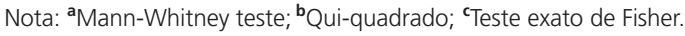
DP: Desvio-Padrão.

Tabela 2. Distribuição trimestral das mulheres em relação aos hábitos de fumo, álcool e atividade física. Campinas (SP), 2011.

\begin{tabular}{|c|c|c|c|c|c|c|c|c|c|c|c|c|c|c|c|c|c|c|c|c|}
\hline \multirow{3}{*}{ Hábitos } & \multicolumn{4}{|c|}{ Basal } & \multicolumn{4}{|c|}{3 meses } & \multicolumn{4}{|c|}{6 meses } & \multicolumn{4}{|c|}{9 meses } & \multicolumn{4}{|c|}{12 meses } \\
\hline & \multicolumn{2}{|c|}{ AMPD } & \multicolumn{2}{|c|}{ DIU } & \multicolumn{2}{|c|}{ AMPD } & \multicolumn{2}{|c|}{ DIU } & \multicolumn{2}{|c|}{ AMPD } & \multicolumn{2}{|c|}{ DIU } & \multicolumn{2}{|c|}{ AMPD } & \multicolumn{2}{|c|}{ DIU } & \multicolumn{2}{|c|}{ AMPD } & \multicolumn{2}{|c|}{ DIU } \\
\hline & $n$ & $\%$ & $n$ & $\%$ & $n$ & $\%$ & $n$ & $\%$ & $n$ & $\%$ & $n$ & $\%$ & $n$ & $\%$ & $n$ & $\%$ & $n$ & $\%$ & $n$ & $\%$ \\
\hline \multicolumn{21}{|c|}{ Tabagismo } \\
\hline Não & 25 & $89,3^{a}$ & 21 & $87,5^{a}$ & 26 & $92,9^{a}$ & 20 & $83,3^{a}$ & 26 & $92,9^{a}$ & 21 & $87,5^{a}$ & 26 & $92,9^{a}$ & 20 & $83,3^{a}$ & 26 & $92,9^{a}$ & 20 & $83,3^{a}$ \\
\hline Sim & 3 & $10,7^{a}$ & 3 & $12,5^{a}$ & 2 & $7,1^{\mathrm{a}}$ & 4 & $16,7^{a}$ & 2 & $7,1^{\mathrm{a}}$ & 3 & $12,5^{a}$ & 2 & $7,1^{\mathrm{a}}$ & 4 & $16,7^{a}$ & 2 & $7,1^{\mathrm{a}}$ & 4 & $16,7^{a}$ \\
\hline \multicolumn{21}{|l|}{ Etilismo } \\
\hline$\leq 1$ dose & 23 & $82,1^{\mathrm{a}}$ & 19 & $79,1^{\mathrm{a}}$ & 22 & $78,6^{\mathbf{b}}$ & 17 & $70,9^{\mathbf{b}}$ & 23 & $82,1^{b}$ & 16 & $66,7^{\mathbf{b}}$ & 21 & $75,0^{\mathbf{b}}$ & 18 & $75,0^{\mathbf{b}}$ & 22 & $78,6^{\mathbf{b}}$ & 18 & $75,0^{\mathbf{b}}$ \\
\hline$>1$ dose & 5 & $17,9^{a}$ & 5 & $20,9^{a}$ & 6 & $21,4^{b}$ & 7 & $29,1^{b}$ & 5 & $17,9^{\mathbf{b}}$ & 8 & $33,3^{\mathbf{b}}$ & 7 & $25,0^{\mathbf{b}}$ & 6 & $25,0^{\mathbf{b}}$ & 6 & $21,4^{b}$ & 6 & $25,0^{\mathbf{b}}$ \\
\hline \multicolumn{21}{|c|}{ Atividade física } \\
\hline Não & 22 & $78,6^{\mathbf{b}}$ & 16 & $66,7^{\mathbf{b}}$ & 20 & $71,4^{\mathbf{b}}$ & 17 & $70,9^{\mathbf{b}}$ & 21 & $75,0^{\mathbf{b}}$ & 17 & $70,9^{\mathbf{b}}$ & 22 & $78,6^{a}$ & 20 & $83,3^{a}$ & 18 & $64,3^{\mathbf{b}}$ & 19 & $79,1^{\mathbf{b}}$ \\
\hline $\operatorname{sim}$ & 6 & $21,4^{b}$ & 8 & $33,3^{\mathbf{b}}$ & 8 & $28,6^{\mathbf{b}}$ & 7 & $29,1^{\mathbf{b}}$ & 7 & $25,0^{\mathbf{b}}$ & 7 & $29,1^{b}$ & 6 & $21,4^{\mathrm{a}}$ & 4 & $16,7^{a}$ & 10 & $35,7^{\mathbf{b}}$ & 5 & $20,9^{b}$ \\
\hline
\end{tabular}

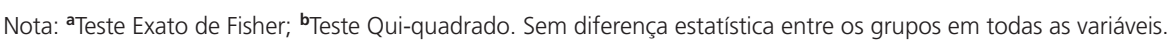

Grupo Acetato de Medroxiprogesterona de Depósito (AMPD, n=28); grupo Dispositivo Intrauterino de Cobre (DIU, n=24).

mostrou mudanças no peso, nas medidas antropométricas e no gasto energético em novas usuárias de AMPD durante o primeiro ano de uso do método. Este é, possivelmente, o primeiro estudo 
Tabela 3. Médias e desvio-padrão trimestrais das medidas antropométricas e gastos energéticos de usuárias de Acetato de Medroxiprogesterona de Depósito (AMPD) e Dispositivo Intrauterino de Cobre (DIU) durante o primeiro ano de uso dos métodos. Campinas (SP), 2011.

\begin{tabular}{|c|c|c|c|c|c|c|c|c|c|c|c|}
\hline \multirow{2}{*}{ Variáveis } & \multicolumn{2}{|c|}{ Basal } & \multicolumn{2}{|c|}{3 meses } & \multicolumn{2}{|c|}{6 meses } & \multicolumn{2}{|c|}{9 meses } & \multicolumn{2}{|c|}{12 meses } & \multirow{2}{*}{$p^{\mathbf{a}}$} \\
\hline & AMPD & DIU & AMPD & DIU & AMPD & DIU & AMPD & DIU & AMPD & DIU & \\
\hline Peso (kg) & $\begin{array}{l}62,4 \\
(9,8)\end{array}$ & $\begin{array}{l}60,5 \\
(6,6)\end{array}$ & $\begin{array}{l}62,3 \\
(9,0)\end{array}$ & $\begin{array}{l}60,3 \\
(6,8)\end{array}$ & $\begin{array}{l}62,7 \\
(9,0)\end{array}$ & $\begin{array}{l}59,9 \\
(6,8)\end{array}$ & $\begin{array}{l}64,1 \\
(9,2)\end{array}$ & $\begin{array}{l}60,3 \\
(6,9)\end{array}$ & $\begin{array}{l}64,6 \\
(9,1)\end{array}$ & $\begin{array}{l}60,3 \\
(7,2)\end{array}$ & 0,221 \\
\hline $\mathrm{IMC}\left(\mathrm{kg} / \mathrm{m}^{2}\right)$ & $\begin{array}{l}23,9 \\
(3,6)\end{array}$ & $\begin{array}{l}24,5 \\
(2,7)\end{array}$ & $\begin{array}{l}23,8 \\
(3,3)\end{array}$ & $\begin{array}{l}24,3 \\
(2,8)\end{array}$ & $\begin{array}{l}24,0 \\
(3,3)\end{array}$ & $\begin{array}{l}24,2 \\
(2,6)\end{array}$ & $\begin{array}{l}24,5 \\
(3,4)\end{array}$ & $\begin{array}{l}24,3 \\
(2,7)\end{array}$ & $\begin{array}{l}24,7 \\
(3,2)\end{array}$ & $\begin{array}{l}24,3 \\
(2,8)\end{array}$ & 0,868 \\
\hline Cintura $(\mathrm{cm})$ & $\begin{array}{c}76,3 \\
(7,6)^{*}\end{array}$ & $\begin{array}{l}77,0 \\
(6,0)\end{array}$ & $\begin{array}{l}75,9 \\
(6,9)\end{array}$ & $\begin{array}{l}76,0 \\
(5,8)\end{array}$ & $\begin{array}{l}76,3 \\
(7,2)\end{array}$ & $\begin{array}{l}74,7 \\
(5,3)\end{array}$ & $\begin{array}{l}77,1 \\
(7,7)\end{array}$ & $\begin{array}{l}75,4 \\
(5,6)\end{array}$ & $\begin{array}{l}77,8 \\
(7,5)\end{array}$ & $\begin{array}{l}75,2 \\
(5,8)\end{array}$ & 0,556 \\
\hline Quadril (cm) & $\begin{array}{c}98,6 \\
(6,7)^{*}\end{array}$ & $\begin{array}{c}100,2 \\
(5,5)\end{array}$ & $\begin{array}{l}99,5 \\
(7,0)\end{array}$ & $\begin{array}{l}99,4 \\
(5,5)\end{array}$ & $\begin{array}{c}100,1 \\
(7,0)\end{array}$ & $\begin{array}{l}99,3 \\
(5,8)\end{array}$ & $\begin{array}{c}101,2 \\
(6,7)\end{array}$ & $\begin{array}{l}99,7 \\
(5,9)\end{array}$ & $\begin{array}{c}102,2 \\
(6,6)\end{array}$ & $\begin{array}{l}99,2 \\
(6,2)\end{array}$ & 0,803 \\
\hline GEB (kcal) & $\begin{array}{c}1337 \\
(249,1)\end{array}$ & $\begin{array}{r}1413 \\
(66,8)\end{array}$ & $\begin{array}{c}1427 \\
(93,3)\end{array}$ & $\begin{array}{r}1405 \\
(73,1)\end{array}$ & $\begin{array}{c}1433 \\
(88,5)\end{array}$ & $\begin{array}{c}1403 \\
(69,2)\end{array}$ & $\begin{array}{c}1443 \\
(87,8)\end{array}$ & $\begin{array}{c}1406 \\
(70,2)\end{array}$ & $\begin{array}{c}1449 \\
(90,6)\end{array}$ & $\begin{array}{c}1403 \\
(71,2)\end{array}$ & 0,295 \\
\hline GET (kcal) & $\begin{array}{c}1738 \\
(323,8)\end{array}$ & $\begin{array}{l}1837 \\
(86,9)\end{array}$ & $\begin{array}{c}1856 \\
(121,3)\end{array}$ & $\begin{array}{c}1826 \\
(95,0)\end{array}$ & $\begin{array}{c}1863 \\
(115,1)\end{array}$ & $\begin{array}{c}1823 \\
(90,0)\end{array}$ & $\begin{array}{c}1812 \\
(335,0)\end{array}$ & $\begin{array}{l}1827 \\
(91,3)\end{array}$ & $\begin{array}{c}1884 \\
(117,2)\end{array}$ & $\begin{array}{l}1824 \\
(92,5)\end{array}$ & 0,330 \\
\hline
\end{tabular}

Nota: análise de Variância: teste para medidas repetidas. *Avaliada 27 mulheres no grupo de AMPD no baseline. Grupo AMPD ( $n=28)$; grupo DIU $(n=24)$.

IMC: Índice de Massa Corporal; GEB: Gasto Energético Basal; GET: Gasto Energético Total.

que avalia usuárias iniciais do contraceptivo a cada trimestre e utiliza grupo de comparação de método não hormonal. As amostras de mulheres distribuídas nos grupos de AMPD e DIU apresentaram características similares, exceto pela maior escolaridade observada no primeiro grupo.

Os resultados corroboram o estudo placebo-controlado, randomizado e cego com 20 mulheres, 10 usuárias de AMPD e 10 usuárias de solução salina, que avaliou o gasto energético basal por meio de calorimetria indireta entre a fase folicular e lútea em um ciclo prévio à administração de AMPD e após administração do injetável. Essa pesquisa não encontrou diferenças no gasto energético basal e no peso entre usuárias de AMPD e de placebo ${ }^{10}$.

Outros estudos prospectivos que utilizaram diferentes métodos para medidas antropométricas, como pregas, circunferências e composição corporal, descreveram variações amplas de peso ${ }^{6,8}$. Estudo com mulheres tailandesas saudáveis em seguimento de 12 meses avaliou a etiologia do ganho de peso e descreveu que 3/12 usuárias de AMPD, ao final do estudo, apresentaram ganho médio de $6 \mathrm{~kg}$ no peso corporal e aumento nas medidas de todas as dobras cutâneas avaliadas ${ }^{8}$. Em pesquisa randomizada com mulheres norte e sulamericanas, europeias e asiáticas comparando o ganho de peso entre duas apresentações de AMPD, intramuscular e subcutânea, realizou-se análise adicional para variações do peso por idade e IMC aos 12 meses de seguimento, descrevendo larga variação individual nas usuárias de AMPD $( \pm 9 \mathrm{~kg}$ em um pequeno número de mulheres e flutuações de $\pm 2,3 \mathrm{~kg}$ na maior parte delas) ${ }^{6}$. Neste estudo não foram detectadas participantes com aumento de peso excessivo, possivelmente devido às orientações sobre alimentação saudável, sobre a importância de exercícios físicos regulares e ao monitoramento de medidas.

Entre as etiologias sugeridas para o ganho de peso em usuárias de AMPD, um estudo internacional descreveu que as mulheres de etnia negra optavam mais frequentemente pelo uso do AMPD, e que o componente étnico poderia justificar o maior ganho de peso apresentado em usuárias do método ${ }^{18}$. Os resultados aqui descritos não concordam com o argumento, pois cerca de dois terços desta amostra eram não brancas e não foi observada diferença no ganho de peso entre os grupos de AMPD e DIU. 
Alguns autores que observaram ganho de peso em usuárias de AMPD sugeriram orientar mulheres que iniciam uso de contraceptivos para hábitos saudáveis de vida, atividade física e dieta $5,6,19,20$. Neste estudo, todas as participantes sabiam do objetivo da pesquisa de avaliar o ganho de peso em mulheres em uso do contraceptivo AMPD. Além disso, a cada retorno, foram estimuladas a ingerir maior quantidade de verduras, leite e derivados e a praticar exercícios físicos aeróbicos regulares três vezes por semana, em especial a caminhada. Acredita-se que essa intervenção possa ter levado a uma maior atenção ao controle do peso, independente do método anticoncepcional utilizado.

Entre os pontos fortes deste estudo figura a amostra de mulheres saudáveis, todas novas usuárias do método, e a avaliação trimestral de medidas realizada sempre pela mesma profissional, a qual também orientava a respeito dos hábitos de vida saudável para manter o peso corporal. Entre as limitações está o tempo de seguimento, o qual foi considerado curto para assegurar a manutenção do peso corporal entre as usuárias de AMPD após o primeiro ano de uso do método. É possível, que um maior tempo de seguimento pudesse detectar diferenças significativas no ganho de peso de usuárias de AMPD, já que o ganho médio nesse grupo foi de $2,2 \mathrm{~kg}$. A falta de randomização também pode sugerir uma limitação, entretanto, acredita-se que houve maior aderência ao uso do método quando a mulher pôde optar pelo contraceptivo, sendo que autores descrevem aumento na taxa de descontinuação quando a escolha ao método é negada ${ }^{21}$. A característica da amostra de usuárias saudáveis impede que esses resultados possam ser generalizados para outras populações, como por exemplo, as portadoras de patologias crônicas.

Assim, mulheres jovens e saudáveis não apresentaram mudança no peso e nas medidas antropométricas no primeiro ano de uso do AMPD. As orientações sobre hábitos de vida saudável, dispensadas regularmente, são impor- tantes para o controle do peso corporal além do monitoramento de medidas antropométricas em mulheres usuárias de métodos contraceptivos hormonais.

\section{A GRADECIMENTOS}

À Coordenação de Aperfeiçoamento de Pessoal de Nível Superior pela bolsa para Priscilla de Nazaré Silva dos Santos.

\section{COLABORADORES}

O projeto foi realizado por A FERNANDES e PNS SANTOS. PNS SANTOS, WO MODESTO, M SIDER e MV BAHAMONDES e participaram da realização da pesquisa e da confecção do artigo. L BAHAMONDES e A FERNANDES realizaram a análise crítica do artigo e todos os autores aprovaram a versão final.

\section{REFER Ê N CIAS}

1. Fraser IS, Weisberg E. A comprehensive review of injectable contraception with special emphasis on depot medroxyprogesterone acetate. Med J Aust. 1981; 1 (1 Suppl.):3-19.

2. Mosher WD, Martinez GM, Chandra A, Abma JC, Willson SJ. Use of contraception and use of family planning services in the United States: 1982-2002. Adv Data. 2004; (350):1-36.

3. Infoplease. Prevalence of contraceptive use in selected countries [cited 2014 Sept 21]. Available from: http://www.infoplease.com/ipa/A0193123. html

4. Pelkman CL, Chow M, Heinbach RA, Rolls BJ. Shortterm effects of a progestational contraceptive drug on food intake, resting energy expenditure, and body weight in young women. Am J Clin Nutr. 2001; 73(1):19-26.

5. Clark MK, Dillon JS, Sowers M, Nichols S. Weight, fat mass, and centraldistribution of fat increase when women use depot-medroxyprogesterone acetate for contraception. Int J Obes. 2005; 29(10): 1252-8.

6. Westhoff C, Jain JK, Milsom I, Ray A. Changes in weight with depot medroxyprogesterone acetate subcutaneous injection $104 \mathrm{mg} / 0.65 \mathrm{~mL}$. Contraception. 2007; 75(4):261-7. 
7. Bakry S, Merhi ZO, Scalise TJ, Mahmoud MS, Fadiel A, Naftolin F. Depot medroxyprogesterone acetate: An update. Arch Gynecol Obstet. 2008; 278(1): 1-12. http://dx.doi.org/10.1007/s00404-007-0 497-z

8. Amatayakul K, Sivasomboon B, Thanangkul O. A study of the mechanism of weight gain in medroxyprogesterone acetate users. Contraception. 1980; 22(6):605-22.

9. Berenson $A B$, Rahman M. Changes in weight, total fat, percent body fat, and central-to-peripheral fat ratio associated with injectable and oral contraceptive use. Am J Obstet Gynecol. 2009; 200(3):329.e1-8. http://dx.doi.org/10.1016/j.ajog. 2008.12.052

10. Pelkman C. Hormones and weight change. J Reprod Med. 2002; 47(9):791-4.

11. Associação Brasileira de Empresas de Pesquisa. Critério de classificação econômica. São Paulo: Abep; 2010 [acesso 2011 maio 2]. Disponível em: http://www.abep.org/novo/Utils/FileGenerate. ash $x$ ?id=46

12. Ford ES, Kohl HW 3rd, Mokdad AH, Ajani UA. Sedentary behavior, physical activity, and the metabolic syndrome among U.S. adults. Obes Res. 2005; 13(3):608-14.

13. World Health Organization. Global recommendations on physical activity for health. Geneva: WHO; 2011.

14. World Health Organization. Physical status: The use and interpretation of anthropometry. Technical Report Series, nº 854. Geneva: WHO; 1995.
15. Frankenfield DC, Muth ER, Rowe WA. The HarrisBenedict studies of human basal metabolism: History and limitations. J Am Diet Assoc. 1998; 98(4):439-45.

16. Isosaki M, Cardoso E, Oliveira A. Manual de dietoterapia e avaliação nutricional: serviço de nutrição e dietética do Instituto do Coração - HCFMUSP. $2^{a}$ ed. São Paulo: Atheneu; 2009.

17. Friendly M. From SAS System for Statistical Graphics. Version 1.2. Cary (NC): SAS Institute Inc.; 1995 (cited 2014 Apr 18]. Available from: http:// www.yorku.ca/SCS/sasmac/fpower.html

18. Vickey Z, Madden T, Zhao Q, Secura GM, Allsworth $J E$, Peipert JF. Weight change at 12 months in users of three progestin-only contraceptive methods. Contraception. 2013; 88(4):503-8. http://dx.doi. org/10.1016/j.contraception.2013.03.004

19. Haider S, Darney PD. Injectable contraception. Clin Obstet Gynecol. 2007; 50(4):898-906.

20. Le YC, Rahman M, Berenson AB. Early weight gain predicting later weight gain among depot medroxyprogesterone acetate users. Obstet Gynecol. 2009; 114(2):279-84. http://dx.doi.org/10.1097/A OG.0b013e3181af68b2

21. Pariani S, Heer DM, Van Arsdol MD Jr. Does choice make a difference to contraceptive use? Evidence from east Java. Stud Fam Plann. 1991; 22(6):384-90.

Recebido: janeiro 5, 2015 Versão final: maio 26, 2015 Aprovado: junho 29, 2015 